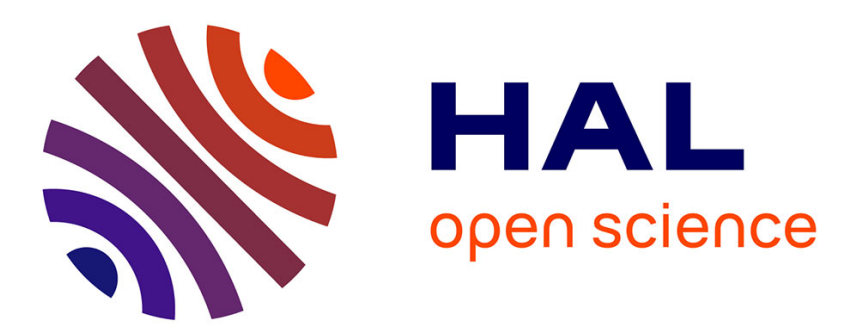

\title{
Large-Eddy Simulation of wind turbines wakes including geometrical effects
}

Pierre Benard, A. Viré, Vincent Moureau, G. Lartigue, L. Beaudet, P. Deglaire, L. Bricteux

\section{- To cite this version:}

Pierre Benard, A. Viré, Vincent Moureau, G. Lartigue, L. Beaudet, et al.. Large-Eddy Simulation of wind turbines wakes including geometrical effects. Computers and Fluids, 2018, 173, pp.133-139. 10.1016/j.compfluid.2018.03.015 . hal-02107334

\section{HAL Id: hal-02107334 \\ https://hal.science/hal-02107334}

Submitted on 26 Nov 2020

HAL is a multi-disciplinary open access archive for the deposit and dissemination of scientific research documents, whether they are published or not. The documents may come from teaching and research institutions in France or abroad, or from public or private research centers.
L'archive ouverte pluridisciplinaire HAL, est destinée au dépôt et à la diffusion de documents scientifiques de niveau recherche, publiés ou non, émanant des établissements d'enseignement et de recherche français ou étrangers, des laboratoires publics ou privés. 


\title{
Large-Eddy Simulation of wind turbines wakes including geometrical effects.
}

\author{
Pierre Bénard ${ }^{1, a}$, Axelle Viré ${ }^{2}$, Vincent Moureau ${ }^{1}$, Ghislain Lartigue ${ }^{1}$, \\ Laurent Beaudet ${ }^{3}$, Paul Deglaire ${ }^{3}$, Laurent Bricteux ${ }^{4}$ \\ ${ }^{1}$ Normandie Univ, INSA Rouen, UNIROUEN, CNRS, CORIA, 76000 Rouen, France \\ ${ }^{2}$ Delft University of Technology, Faculty of Aerospace Engineering, \\ Kluyverweg 1, 2629HS Delft, The Netherlands \\ ${ }^{3}$ Adwen Offshore, INSA Rouen, 76801 Saint-Etienne-du-Rouvray, France \\ ${ }^{4}$ Université de Mons (UMONS), Polytechnic Faculty, Belgium \\ ${ }^{a}$ Corresponding author: pierre.benard@coria.fr \\ Preprint version*
}

\begin{abstract}
Accurate simulation of wind turbine wakes is critical for the optimization of turbine efficiency and prediction of fatigue loads. These wakes are three-dimensional, complex, unsteady and can evolve in geometrically complex environments. Modeling these flows calls thus for high-quality numerical methods that are able to capture and transport thin vortical structures on an unstructured grid. It is proposed here to assess the performances of a fourth-order finite-volume LES solver to perform massively parallel scale-resolving simulations of wind turbines wakes. In this framework, the actuator line method that takes the effect of the wind turbine blades on the flow into account is implemented. It is demonstrated that both near and far parts of the turbine wakes are accurately modeled as well as geometrical details. The methodology is assessed on two different test cases and validated with experimental results. It is demonstrated that the flow predictions are of equivalent quality on both structured and unstructured grids. The influence of the geometrical details (e.g. nacelle and tower) on the wake development as well as the influence of the discretization scheme are also investigated.

Keywords: wind turbine ; wakes ; Large-Eddy Simulation ; actuator line
\end{abstract}

\section{Introduction}

Considering current energetic and environmental challenges, wind turbines constitute an interesting alternative source of energy. It is indeed a renewable source of energy that helps reducing greenhouse gas emissions, by replacing combustion systems such as gas turbines or coal furnaces. In order to provide a large amount of electric power and reduce costs, wind turbines are usually gathered in the same location called a windfarm. Maximizing the electrical power yielded by a windfarm is currently a key issue [1]. For that purpose, the placement of the wind turbines with respect to each other has to be optimized. As the topology of the turbulent vortical wakes emanating from the tip of the blades can have a significant impact on the performances and the mechanical fatigue of the wind turbines located downstream, it is important to provide realistic modeling of these wakes. The latter are vortical flows which are fully turbulent: their simulation thus requires high-quality numerics with both low dispersion and diffusion errors to be able to transport them on long distances without artificial distortion. The range of space and time scales of the turbulence is so wide in such configurations that a direct numerical simulation (DNS) is not affordable. This calls for Large-Eddy Simulation (LES) turbulence modeling. LES is indeed well adapted to this problem since the considered flows are 3D, complex and strongly unsteady. As fully-resolved LES including a moving mesh discretizing the rotor is still expensive, aerodynamics models are often used to represent the effect of the rotating blades on the flow. There are many studies in the literature that are devoted to wind turbine rotor modeling. Most common methods used to model the effect of the rotating blades on the flow are actuator disc and actuator line methods. Actuator disc methods have been extensively used by several authors and results agree fairly well with that of experiments. Actuator disk methods were used in early LES studies of wind turbines wakes (see 2, 3]) and are still used in the context of large-scale windfarms simulations. An original and computationally efficient approach was developed by

*Accepted for publication in Computers \& Fluids. This is a preprint version, the final version can be retrieved at https://doi.org/10.1016/j.compfluid.2018.03.015 
Chatelain et al. (see [4, 5]) who coupled a vortex particle-mesh method with immersed lifting lines for the Large-Eddy Simulation of wind turbine wakes. This method was successfully applied to the study of large-scale aerodynamics and wake behavior of horizontal and vertical axis wind turbines. This method is very well suited for free wake unbounded problems, however, it does not allow for complex geometries. In the framework of Eulerian discretizations, the actuator line method [6] is currently the most advanced model for the rotating blade aerodynamics [7].

In this paper, we propose to demonstrate that a massively parallel high-order finite-volume unstructured flow solver with an actuator line model is able to provide accurate flow predictions of wind turbine wakes. We first investigate the suitability of using fully unstructured meshes to simulate wind turbine wake flows. To this aim, the wake of a generic wind turbine similar to the Tjaereborg wind turbine is simulated [8, 9].

The other test case considered here is the NTNU wind tunnel model [10. There is a great amount of experimental data available on this benchmark, that will allow validating the proposed approach. In particular, the geometrical effect of the tower and nacelle on the wake will be taken into account and its influence will be demonstrated using wall-modeled LES.

\section{Numerical method}

\subsection{Governing equations}

The governing fluid dynamics equations are the Navier-Stokes equations for incompressible flow, supplemented by a subgrid-scale (SGS) model. The filtering operator, which consists in projecting a field on the LES grid, is written as $\widetilde{\bullet}$. Using this notation, the evolution equations for the LES velocity field are formally written as:

$$
\begin{aligned}
\nabla \cdot \widetilde{\mathbf{u}} & =0 \\
\frac{\partial \widetilde{\mathbf{u}}}{\partial t}+(\widetilde{\mathbf{u}} \cdot \nabla) \widetilde{\mathbf{u}} & =-\nabla \widetilde{P}+\nu \nabla^{2} \widetilde{\mathbf{u}}+\nabla \cdot \widetilde{\boldsymbol{\tau}}^{M}+\mathbf{f},
\end{aligned}
$$

where $\widetilde{P}=\frac{1}{\rho} \widetilde{p}$ is the reduced pressure field, $\nu$ the kinematic viscosity, and $\widetilde{\tau}^{M}$ the modeled SGS stress tensor. The body force $\mathbf{f}$ is introduced to model the effect of the wind turbine rotor onto the flow.

\subsection{YALES2 flow solver}

The Navier-Stokes solver YALES2 [11] is used here to solve Eqs. 1 and 2, YALES2 is a massively-parallel finite-volume solver using a central 4th-order numerical scheme for spatial discretizarion, and a 4th-order 2step Runge-Kutta method [12] for the time integration. The discretization is strictly fourth-order on Cartesian grids. On regular tetrahedral grids, i.e. with a homogeneous control volume size, the spatial errors are fourthorder but this order of convergence can decrease on low quality grids as classical finite volumes do. This code solves the incompressible and low-Mach number Navier-Stokes equations for turbulent flows on structured or unstructured meshes using a projection method for pressure-velocity coupling [13. The Poisson equation in the projection method is solved with a high-performance Deflated Preconditioned Conjugate Gradient solver as described in [14. Combined with an in-house hierarchical grid partitioning, the code can handle grids with several billions of elements [15].

\subsection{Actuator line method}

Due to the multi-scale nature of the problem, resolving simultaneously turbine blade boundary layers and vortical wakes over long distances is not computationally affordable. An actuator line method allows to model the influence of the wind turbine on the flow by imposing body forces distributed along rotating lines representing the aerodynamic loads of the wind turbine blades (see e.g. Sorensen et al. [16], Troldborg [17, Peet et al. [18]). This technique also avoids handling moving mesh for the blades. In an actuator line model, the blades are divided into elements that discretize the blade span and have a prescribed rotational motion. Following the formalism used in [18, the local lift $L$ and drag $D$ forces experienced by each element are computed as

$$
\begin{aligned}
L & =\frac{1}{2} \rho v_{r e l}^{2} c(r) w C_{L}(\alpha), \\
D & =\frac{1}{2} \rho v_{r e l}^{2} c(r) w C_{D}(\alpha),
\end{aligned}
$$

where $C_{L}$ and $C_{D}$ are the lift and drag coefficients, $\alpha$ is the local angle of attack, $V_{\text {rel }}$ is the local velocity relative to the rotating blade, $c(r)$ is the local chord length, and $w$ is the actuator element width. The relative velocity is obtained from the velocity triangle depicted in Fig. 1 .

$$
v_{r e l}=\sqrt{v_{z}^{2}+\left(\Omega r-v_{\theta}\right)^{2}}
$$


The local angle of attack $\alpha$ is computed as

$$
\alpha=\phi-\beta=\arctan \left(\frac{v_{z}}{\Omega r-v_{\theta}}\right)-\beta(r),
$$

where $\phi$ is the angle between the relative velocity $v_{r e l}$ and the rotor plane while $\beta(r)$ is the local pitch angle varying along the blade span. In this study, the variations of $C_{L}$ and $C_{D}$ with the angle of attack, as well as pitch and chord variations along the blade, are modeled using curve fitting methods. This strategy avoids making use of look-up tables. The body force source term is applied on the flow using a mollifying function $\eta$ (see e.g. [19, 16]) that provides a smooth force distribution $\mathbf{f}$ obtained from the forces concentrated on a given blade element $e$ as depicted in Fig. 2 .

$$
\mathbf{f}(x, y, z, t)=-\sum_{e=1}^{N}\left(L \mathbf{e}_{L}+D \mathbf{e}_{D}\right) \eta\left(\left|\mathbf{r}-\mathbf{r}_{e}\right|\right) .
$$

The mollifier is defined as

$$
\eta(d)=\frac{1}{\epsilon^{3} \pi^{3 / 2}} \exp \left[-\left(\frac{d}{\epsilon}\right)^{2}\right] .
$$

As in 9], the regularization parameter $\epsilon$ is set to $\epsilon=2 \delta$, where $\delta$ is the actuator line element size. In order to compute $v_{r e l}$, the local velocity components are interpolated from the grid to the actuator line elements using a linear interpolation scheme.

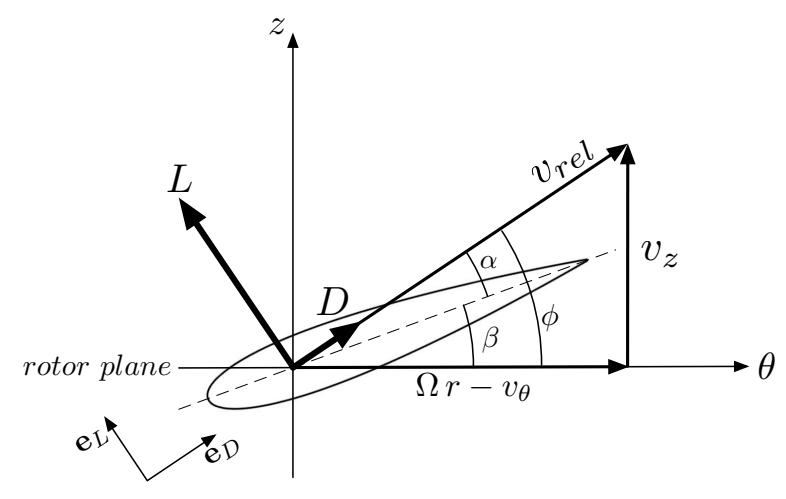

Figure 1: Velocity triangle and geometrical conventions.

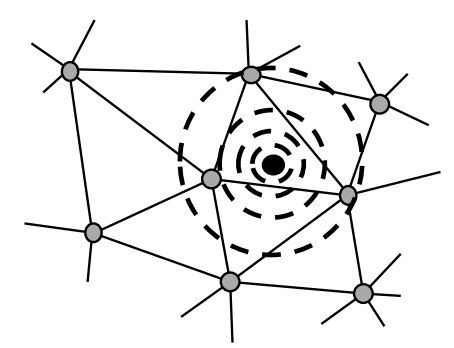

Figure 2: Mollification of a point force on several nodes of an unstructured grid.

\subsection{Subgrid scale and wall modeling}

In this study, the SGS $\sigma$-model developed by Nicoud et al. 20] is used. In this model, the turbulent viscosity vanishes as soon as the resolved field is either two-dimensional or two-component. It is also inactive in pure shear and solid rotation flows. Moreover, the subgrid scale viscosity $\nu_{s g s}$ vanishes with the appropriate theoretical $y^{+^{3}}$ behavior in the vicinity of solid boundaries. As stated in [20, the $\sigma$-model provides results of similar quality at a lower cost compared to the classical dynamic Smagorinsky model. Due to the excessive computational cost needed to properly capture turbulent boundary layers developing along solid walls at high Reynolds number, wall resolved LES cannot be performed here. Thus, we use wall-modeled LES that resolves the energetic eddies in the outer layer and models all the eddies in the inner layer. The inner layer dynamics is thus modeled by imposing a wall shear stress $\tau_{w}$ computed here with a standard log-law wall model. This model is used only on the non-rotating parts of the wind turbine, i.e. on the tower and nacelle. 


\section{LES of a generic $30 \mathrm{~m}$ rotor horizontal axis wind turbine: struc- tured vs. unstructured grid}

The first case investigated to assess the proposed method is representative of the Tjaereborg [ $[8,9$, wind turbine. It has a diameter $D=2 R \approx 61 \mathrm{~m}$ and the rotor blade profiles are modeled using NACA4418 airfoils as in [18], with a chord length decreasing linearly from hub to tip. The wind speed $U_{\infty}$ and the rotational speed $\Omega$ are set so that the tip speed ratio $T S R=\lambda=\frac{\Omega R}{U_{\infty}}=7.07$. The aim of this investigation is to validate the fact that the numerical method proposed for unstructured grids is able to provide results of equivalent quality compared to a fully Cartesian structured grid.

\subsection{Computational setup}

Taking the rotor diameter $D$ as reference length scale, the lengths of the computational domain are $L_{x}=L_{y}=$ $9 D$ and $L_{z}=15 D$ as indicated by Fig. 3 . The number of grid points for the structured grid are $N_{x}=N_{y}=256$
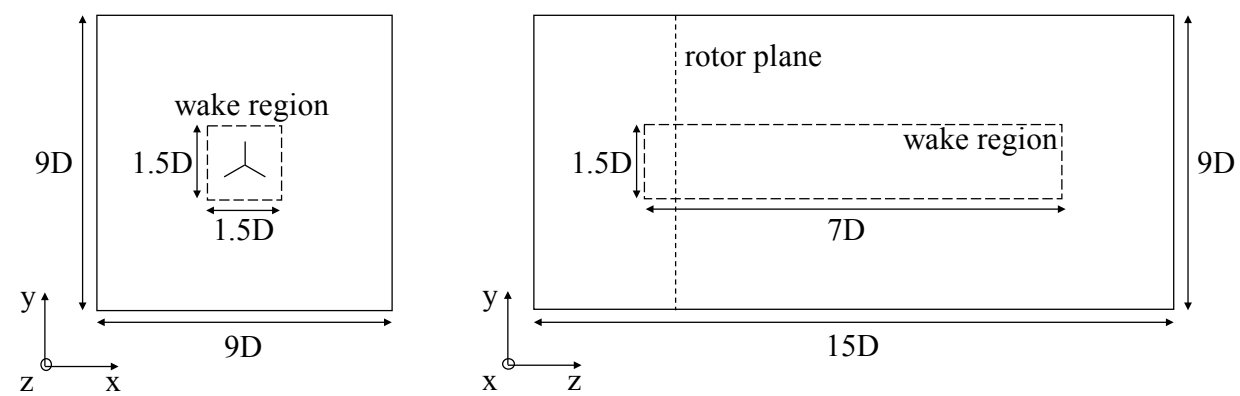

Figure 3: Description of the Tjaereborg turbine geometry and computational domain.

and $N_{z}=960$, leading to a total of 63 millions nodes. The structured grid is stretched so that the cells in the wake and rotor regions have a uniform size distribution and are relatively coarse in irrotational regions. The unstructured grid is built to have a similar cell size in the rotor and wake regions to the structured mesh (about $h=0.08 D$ ), and progressively coarsens outside. It includes almost 50 millions nodes. All the computations of this section are performed with a fourth-order spatial discretization.

\subsection{Flow Visualization}

Figure 4 allows the observation of the vorticity tubes generated along the blades. These helical vortical structures are generated at the rotor tip and hub where the circulation gradient is the highest. Each helical structure is subjected to growing instabilities and further transition to a fully turbulent flow after a distance of approximately $1 D$. This flow visualization also demonstrates the ability of the proposed method to capture large eddies as well as finer turbulence structures over a long distance. The hub vortex is also well captured. It exhibits a regular helical pattern in the rotor region and quickly transition to turbulence also $1 D$ downstream.

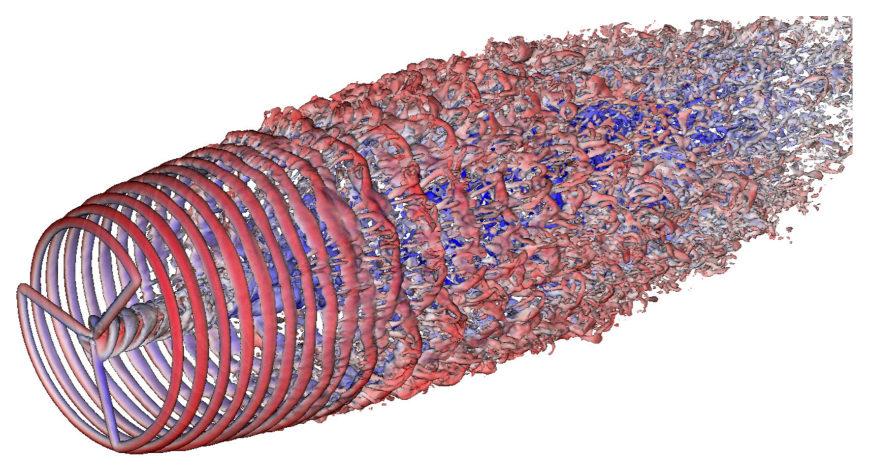

Figure 4: Visualization of the flow field using isosurfaces of vorticity norm $\|\vec{\omega}\|$.

\subsection{Flow diagnostics}

Flow statistics accumulated for 50 rotor rotations are compared thanks to the induction factor $a=1-\left\langle u_{z}\right\rangle / U_{\infty}$ and the turbulent kinetic energy (TKE) profiles at three positions downstream the rotor in Fig. 5 . We can 
conclude that the results obtained using an unstructured grid are of equivalent quality compared to those obtained on a structured grid of equivalent resolution. Induction factor profiles are almost coincident even $5 D$ downstream. TKE profiles are also almost identical, except in the region outside the wake $r / R>1$. These slight discrepancies may be due to the coarsening of the unstructured grid outside of the wake zone.
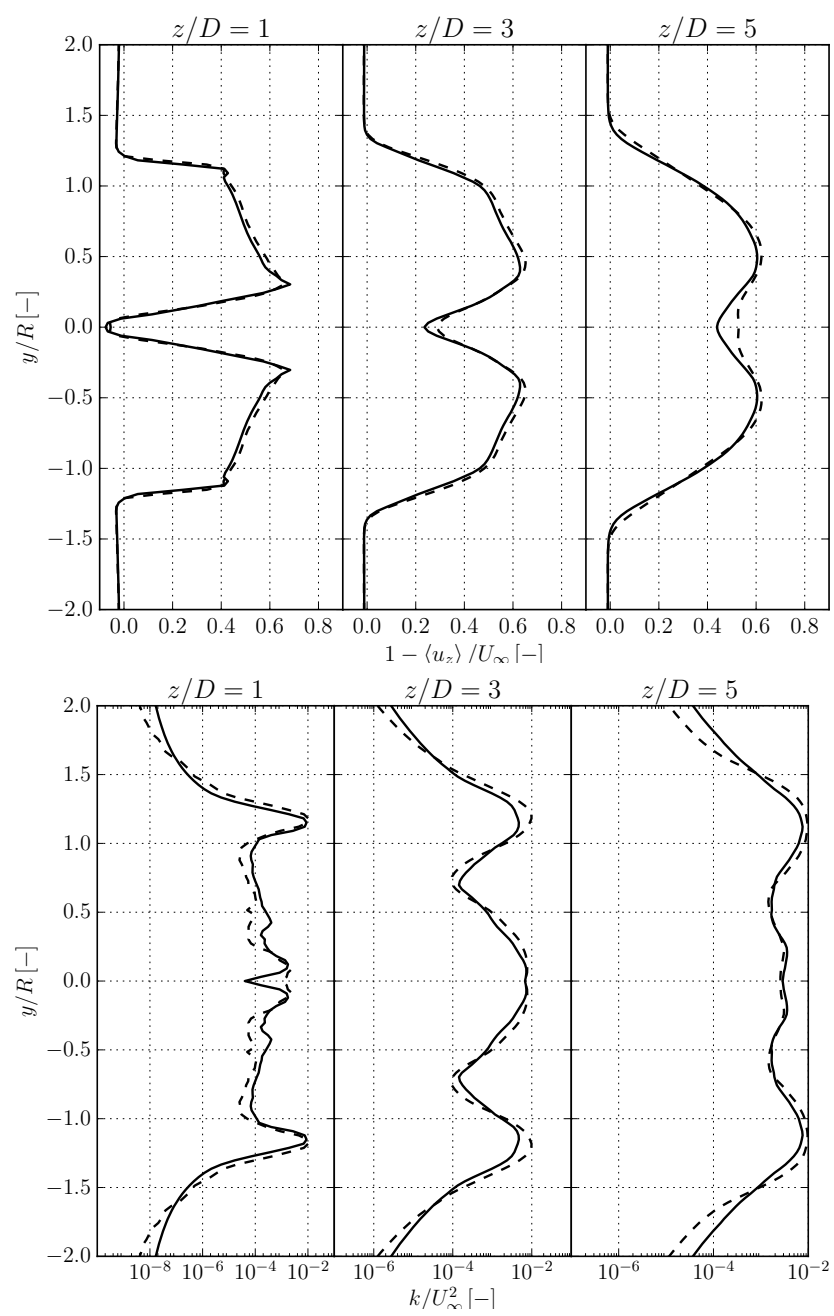

Figure 5: Induction factor (top) and turbulent kinetic energy (bottom), LES results on structured (solid), LES results on unstructured grid (dash).

\subsection{Grid convergence study on unstructured mesh}

A grid convergence study was performed to assess the mesh resolution needed to properly capture the wake dynamics. Three unstructured grids were tested whose properties are summarized in Tab. 1 . A first mesh M1 was generated with 0.77 million points and a cell size in the wake region of $h=0.031 D$. Then, two homogeneous grid refinements (thanks to the method of Rivara 21]) are applied to produce the M2 and M3 grids. The induction factor and TKE profiles at three positions downstream the rotor are plotted in Fig. 6

\begin{tabular}{lcccc}
\hline Mesh & \# nodes & $h / D$ & $\Delta t[\mathrm{~ms}]$ & \# elemblade \\
\hline M1 & $0.77 \times 10^{6}$ & 0.031 & 112 & 14 \\
M2 & $6.00 \times 10^{6}$ & 0.015 & 31.1 & 28 \\
M3 & $49.3 \times 10^{6}$ & 0.008 & 15.3 & 57 \\
\hline
\end{tabular}

Table 1: Grid characteristics for the Tjaereborg case.

Differences in induction factor can only be observed behind the hub position in the near-wake region, where the grid M1 shows a strongest blockage of the flow. The TKE presents more discrepancies between meshes. M1 seems to capture much less flow dynamics compared to M2 and M3. These two meshes show good agreement 
as the distance from the rotor increases. These results point out the need to have a sufficient grid resolution and an actuator line discretization of $\delta \simeq h \leq 0.015 D$.
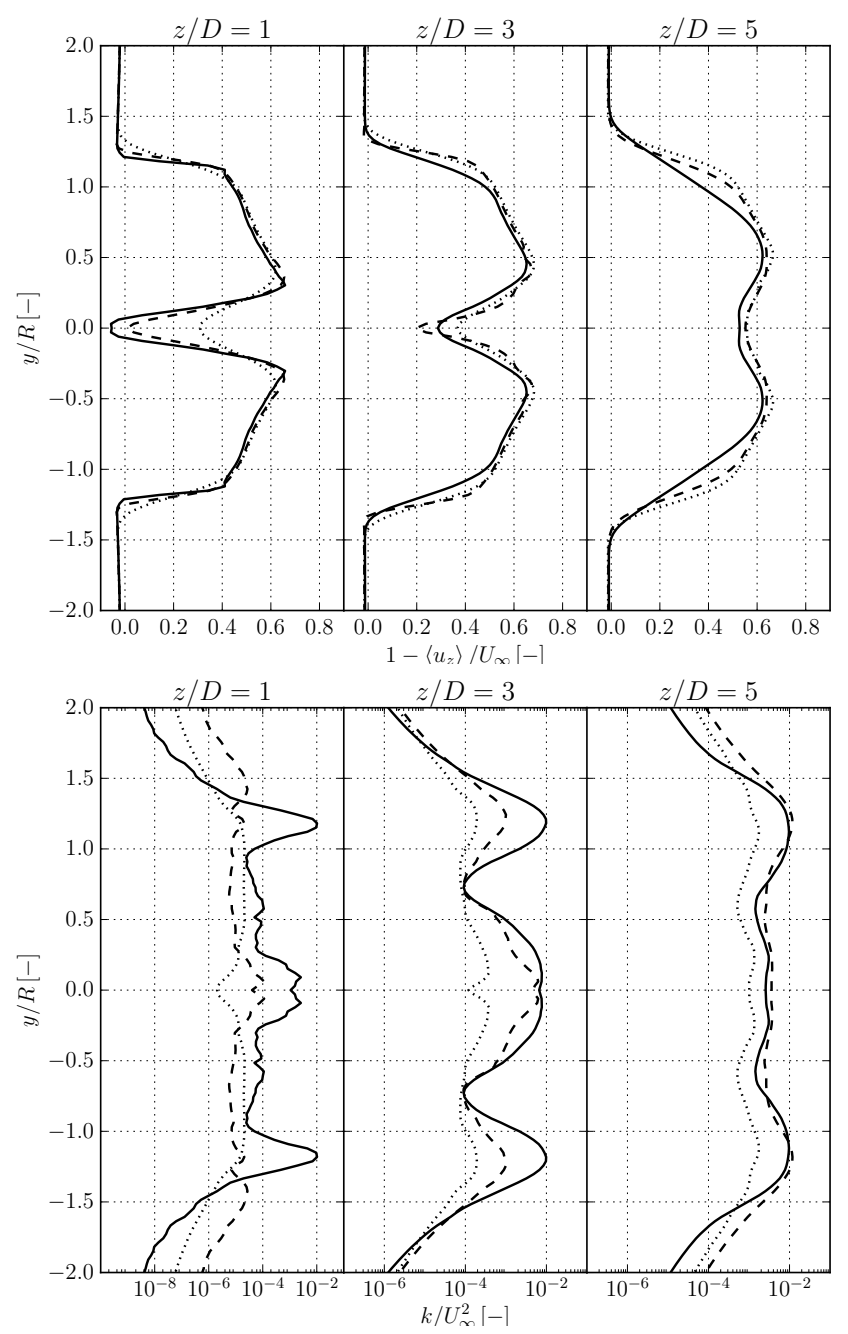

Figure 6: Induction factor (top) and turbulent kinetic energy (bottom), LES results on unstructured grid with coarse M1 (dotted), intermediate M2 (dashed) and fine M3 (solid) grids.

The CPU time needed to perform 50 rotations is 12,290 and 6465 hours on grids M1, M2 and M3, respectively, with 16, 56 and 392 cores. A ratio of 16 is expected at each refinement step: a factor of 2 in each space direction plus an additional factor of 2 due to the time step reduction to keep a constant CFL. It should be noted that other stability constraints due to viscous terms for instance are not limiting in the present cases. It is observed that this ratio is 24 between M1 and M2, indicating a modification of the flow dynamics: higher velocity peaks are observed on M2, which imply a smaller time step to remain below of the CFL constraint (see Tab. 1). Between M2 and M3, the flow dynamics is similar so the time step is reduced by a factor of 2 as expected but the MPI parallel communications between CPUs becomes significant leading to a higher CPU computational time. This is a well-known issue of low-Mach number projection methods, in which the solving of the Poisson equation limits the weak scaling.

\section{LES of a model wind turbine located in a wind tunnel}

The test case investigated here was described in detail in the report by Krogstad et al. [10]. This report provides geometrical parameters, wind tunnel inlet condition and CAD files for the blades and nacelle. The main focus of the proposed blind test was on the wake development behind the turbine. We propose here to assess the validity of our method and highlight the effects of the geometrical details as well as the order of the numerical scheme. 


\subsection{Test case description}

The wind turbine model considered here consists in a three bladed rotor (diameter $D=2 R=0.894 \mathrm{~m}$ ) connected to a nacelle and a tower placed in a wind tunnel. The blade profile is a NREL S826 airfoil. The dimensions of the wind tunnel are such that $L \times W \times H=13.42 D \times 3.35 D \times 2.24 D$, as reported in Fig. 7. The chord

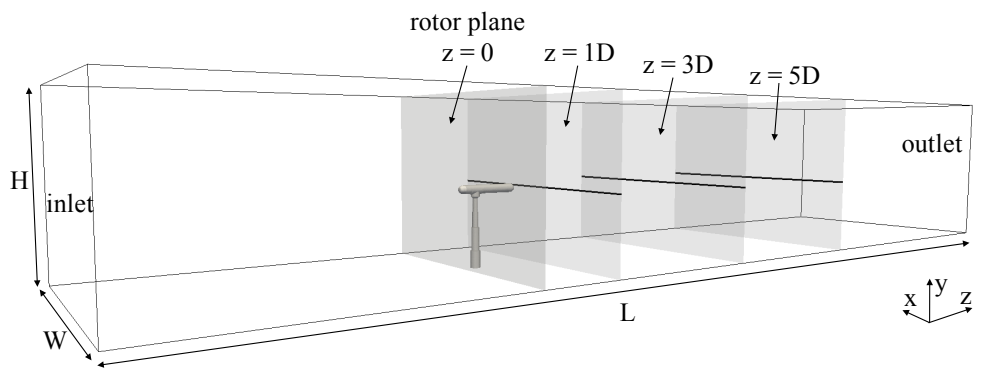

Figure 7: Visualization of the computation domain of NTNU test case with tower and nacelle description. The three back lines refer to the experiment measurements.

length $c(r)$ and pitch angle distribution $\beta(r)$ are reported in Fig. 8. An analytical curve fitting model has been derived for $c, \beta, C_{L}$ and $C_{D}$ in order to avoid the use of lookup tables (see Fig. 9). These curve fitting models consist in trigonometric expansions blended with tanh functions to make the fit vanish when experimental data are not available. The wind speed is uniform and set to $U_{\infty}=10 \mathrm{~m} / \mathrm{s}$ and the rotational speed $\Omega$ is such that $T S R=\lambda=\frac{\Omega R}{U_{\infty}}=6$.
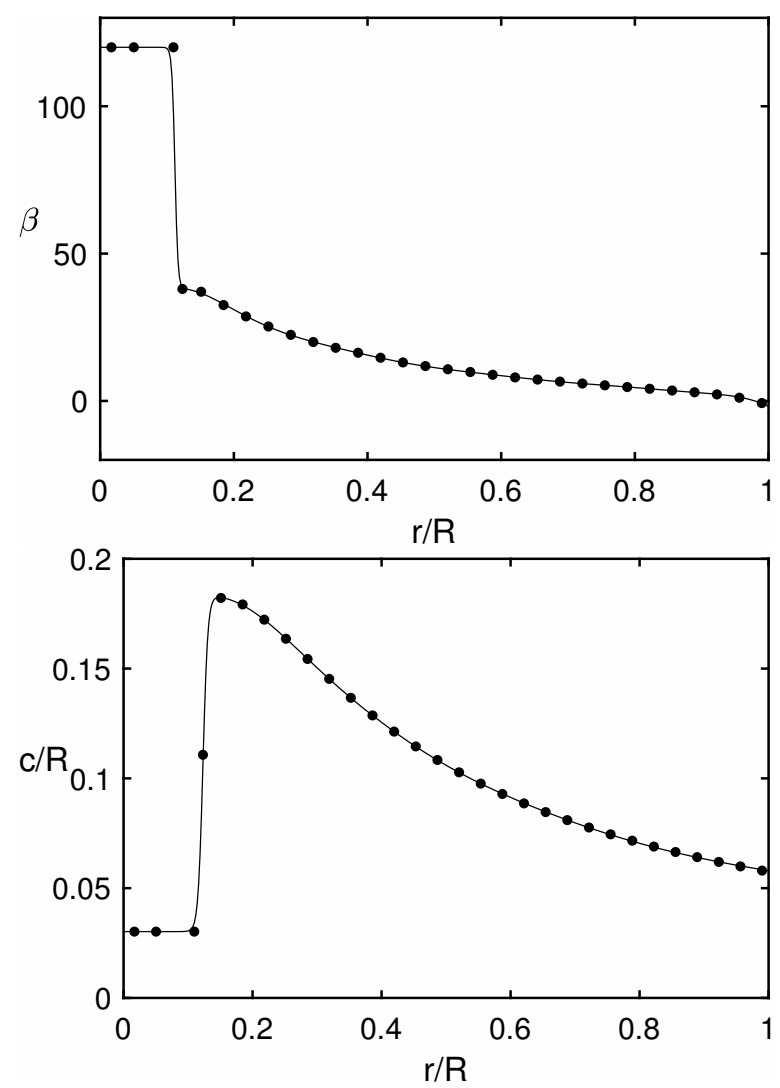

Figure 8: Radial distribution of the pitch angle (top) and chord (bottom). Solid lines show analytical curve fitting model, bullets show data provided in [10].

\subsection{Computational setup}

The grid used here is an unstructured grid including $130 \times 10^{6}$ tetrahedral cells and $22 \times 10^{6}$ nodes with $h \approx 0.015 D$ in the rotor zone. The use of an unstructured grid allows to take into account tower and nacelle geometrical effects. It also allows to use a refined mesh in rotor and wake zones as depicted in Fig. 10. Blades 

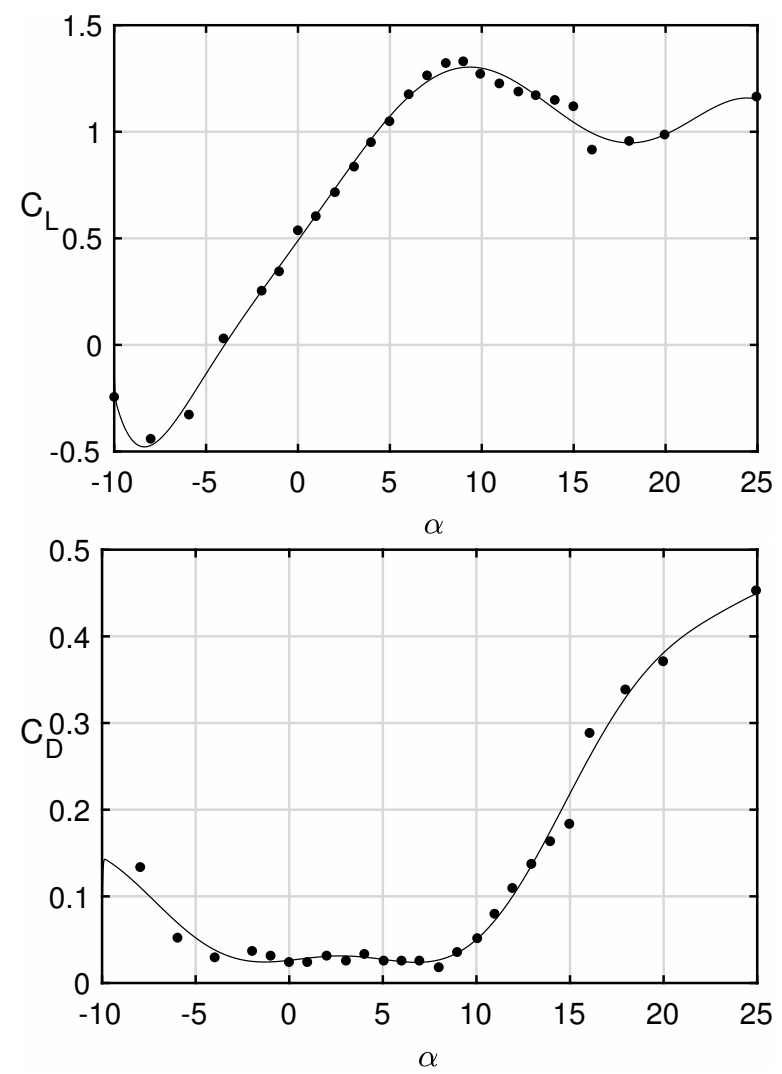

Figure 9: Lift coefficient (top) and drag coefficient (bottom). Solid lines show curve fitting model, bullets show data provided in [10].

are modeled thanks to 32 particles in the actuator line, equally distributed on the blade radius, whose body forces are displayed in Fig. 11 .

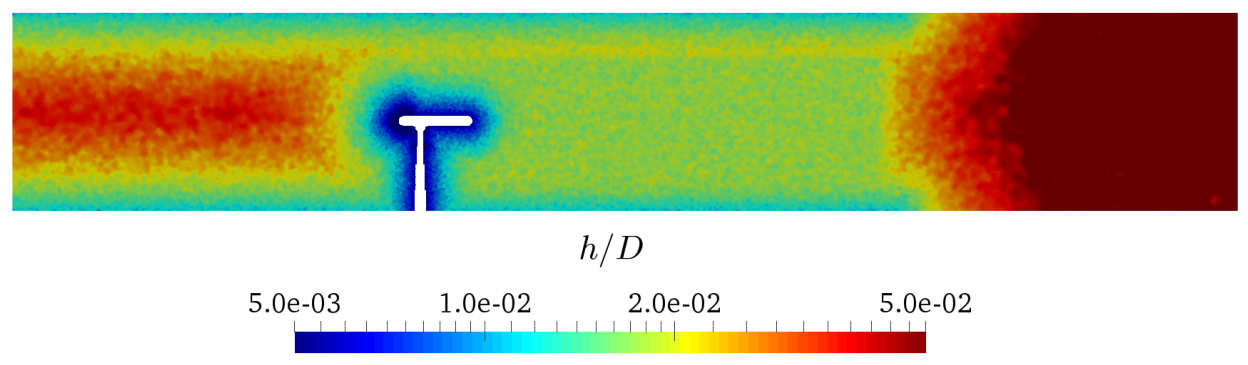

Figure 10: Side view (top) of the unstructured grid size used to capture geometrical details of nacelle and tower with the refinement box.

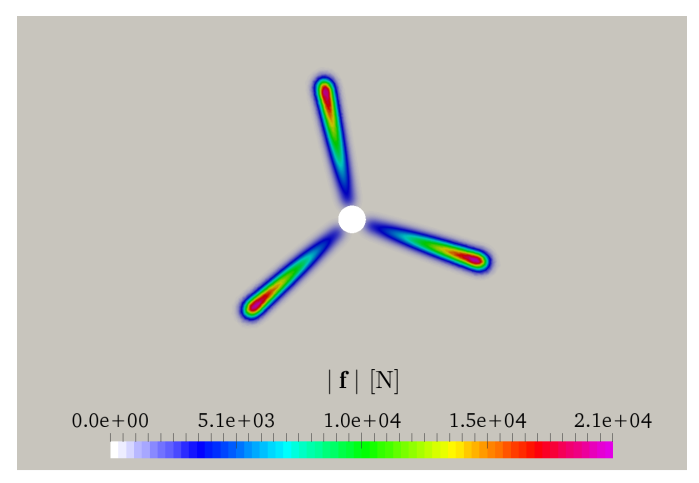

Figure 11: Norm of the actuator line body force applied by the blades on the flow in the rotor plane. 


\subsection{Tower and nacelle effects}

It is proposed here to compare the results obtained with and without considering tower and nacelle effects. Two LES are performed, one with a simple computational box, including only the effect of the wind tunnel walls, and one including the detailed geometry of the wind turbine (see Fig. 10). Both grids are unstructured using tetrahedral elements. Profiles are compared to experimental measurements at three positions downstream the rotor (see Fig. 7). The velocity and TKE profiles displayed in Fig. 12 are significantly different when geometrical effects are taken into account. A profile asymmetry is observed when tower and nacelle are modeled and a better agreement with experimental results is also clear. Considering the induction factor, it can be seen that the nacelle has a significant effect on the velocity deficit. Indeed, in the near wake region $(x / D \approx 1)$, when $y / R \approx 0$, the velocity deficit is much higher due to the presence of the nacelle and the value predicted by the LES is closer to that of the experiment.
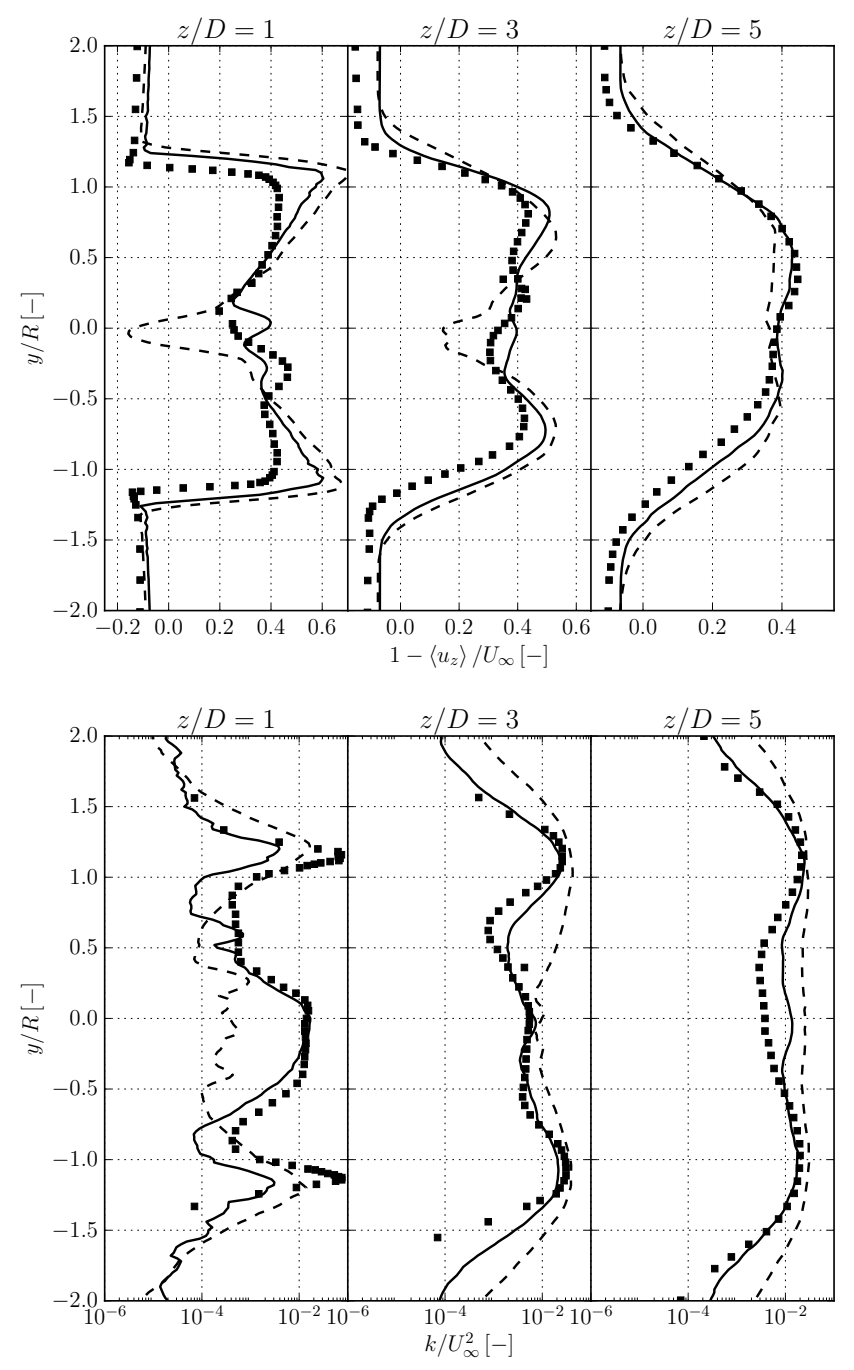

Figure 12: Induction factor (top) and turbulent kinetic energy (bottom), experimental data (square), LES results with nacelle and tower effects (solid), LES results without details (dash).

\subsection{Effect of the spatial discretization order}

In order to show that the proposed approach performs better than classical low-order finite-volume methods on unstructured grids, it is proposed to investigate the performances of three different spatial discretization schemes. The RK4 time integration scheme is not changed. The first considered scheme is a first-order upwind standard finite-volume method. This first-order scheme is supposed to emulate what is obtained using a classical finite-volume scheme on an unstructured grid including tetrahedral elements. The second and third schemes are respectively second- and fourth-order accurate in space on both structured and unstructured grid provided that the cell size distribution is homogeneous in the region of interest. It is interesting to compare the ability of these schemes to properly capture vortical wakes emanating from the rotor. Figure 13 shows the vorticity 
field produced using the three different schemes. As depicted in Fig. 13, the performances of the first-order scheme are quite poor. The helical vortical structure that is usually observed experimentally downstream the rotor is merged due to numerical diffusion into a cylindrical shear layer with much decayed circulation. After one diameter downstream, the wake has almost completely disappeared. This scheme, that is representative of a classical finite-volume scheme on an unstructured grid, is thus not able to reproduce properly the wake vorticity dynamics. For the second-order scheme, the helical structure of the wake is quickly dissipated. The vortices issued from the tip are merged with the creation of a cylindrical shear layer that eventually becomes unstable. However, it can be observed that the vorticity peak decays due to numerical diffusion effects that alter significantly the wake dynamics. The tip vortex tube identity is preserved longer using the fourth-order scheme. The vortical structures in the wake of the nacelle are diffused later compared to the results provided by the second-order scheme. This qualitative analysis shows that the fourth order scheme used here allows to better preserve the wake dynamics compared with the two other schemes that are more representative of what classical finite-volume solvers would give.

The induction factor and TKE profiles reported in Fig. 14 show the same trends. Indeed, the fourth-order scheme produces profiles that are consistent with the experimental data.

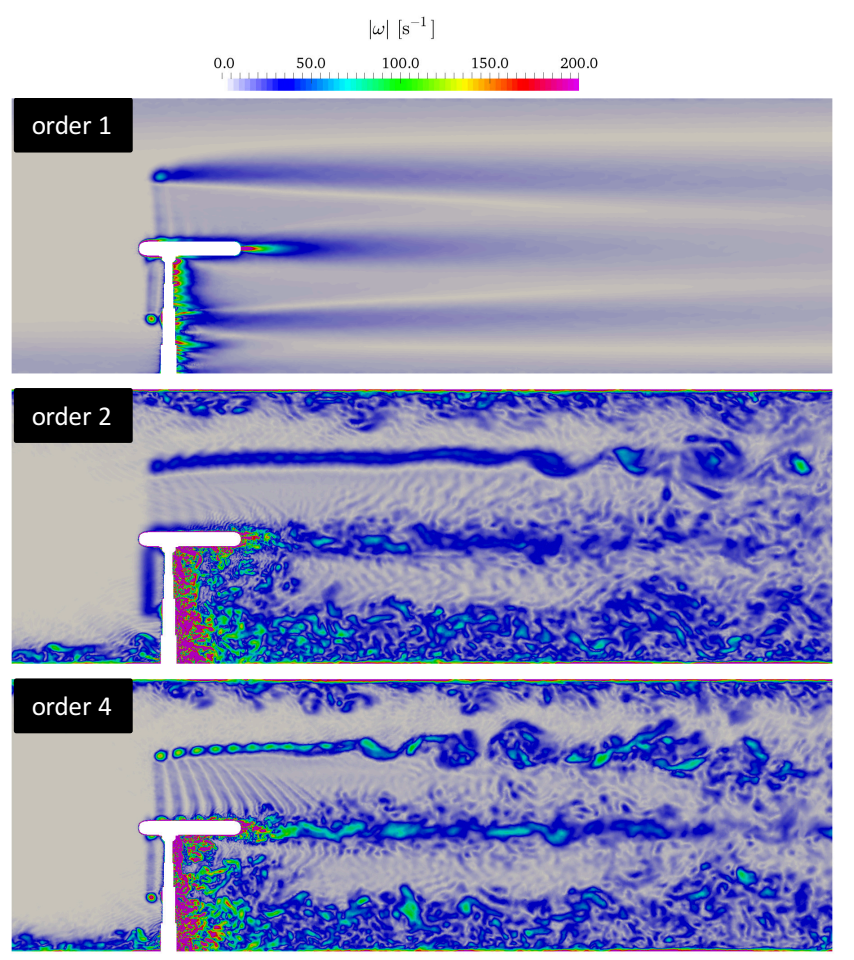

Figure 13: Visualization of the flow field using iso-countours of vorticity norm $\|\vec{\omega}\|$.

The computational time needed for one rotation of the rotor and the Reduced Computational Time (global CPU time per control volume and per time step) for each case are reported in Tab. 2. The three cases show very similar computing performances as the computing time is mainly piloted by the Poisson solver efficiency and not by the spatial discretization order. With its negligible impact on the simulation return time, the fourth-order spatial scheme can always be considered as the best choice.

\begin{tabular}{lcc}
\hline Spatial order & $t_{C P U} /$ rotation $[\mathrm{h}]$ & $\mathrm{RCT}[\mu s]$ \\
\hline 1 & 128 & 41.0 \\
2 & 125 & 43.6 \\
4 & 134 & 46.0 \\
\hline
\end{tabular}

Table 2: Computing performances of NTNU cases with geometrical details.

\section{Concluding Remarks}

This work demonstrates the ability to perform massively parallel scale-resolving simulations where both near and far turbine wakes, as well as geometrical details, are accurately modeled. Thanks to the use of unstructured 

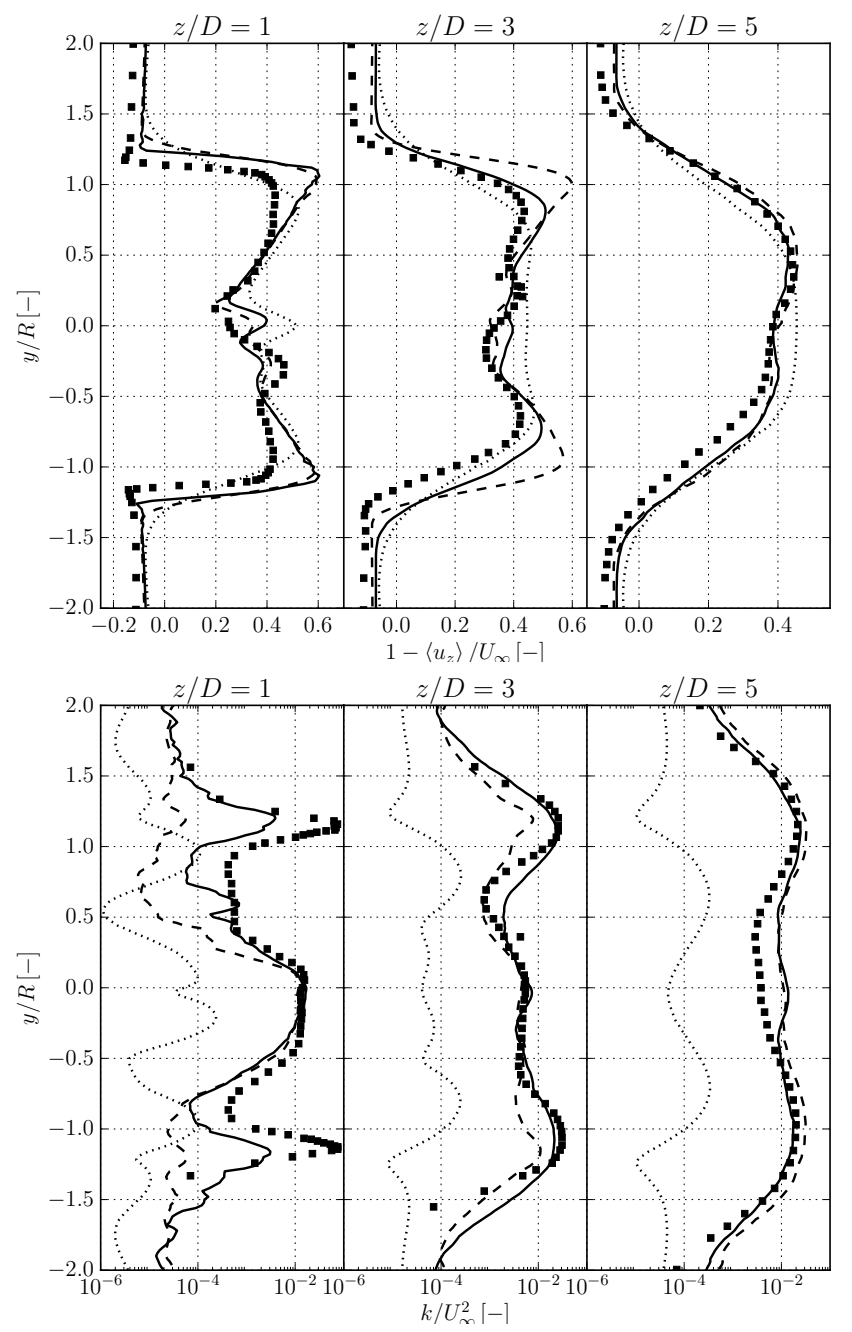

Figure 14: Induction factor (top) and turbulent kinetic energy (bottom), experimental data (square), fourth order scheme (solid), second order (dash), first order upwind (dots).

grids, complex geometries can be handled. This was assessed by considering tower and nacelle effects on a well-known yet severe benchmark case.

These simulations can be further exploited to get more insights into wind turbine wakes and their interactions with complex environments and to validate design tools based on simpler models. Moreover, it has been demonstrated that the computations on unstructured grids provide results of the same quality as those obtained on structured grids. The spatial discretization scheme used has a great influence on the wake development, showing that here, schemes that are at second- or first-order accurate on unstructured grids provide poor performances for the prediction of the wake development. Higher-order schemes with low dissipation and dispersion present a clear advantage for a given grid.

Many further investigations can be carried with the proposed methodology. The actuator line model can be improved, the effect of complex terrain and buildings can be considered... Thanks to the interface of YALES2 with the mesh generation library MMG [22], automatic mesh refinement in the wake can be easily incorporated and assessed.

\section{Acknowledgments}

This work was granted access to the HPC resources from CINES (Centre Informatique National de l'Enseignement Superieur), from IDRIS (Institut du Developpement et des Ressources en Informatique Scientifique) and from TGCC-CEA under the allocations x20172b6880 made by GENCI (Grand Equipement National de Calcul Intensif). It was also granted CPU time by CRIANN under the allocation 2012006. This work is co-financed by the European Union with the European regional development fund (ERDF, HN0002137) and by the Normandie Regional Council via the M2NUM project. P.B., G.L, V.M., L.Be. and P.D. are also supported by the joint project INWIT funded by INSA Rouen Normandie, Adwen Offshore and Région Normandie. The present research also benefited from computational resources made available on the Tier-1 supercomputer of 
the Fédération Wallonie- Bruxelles, infrastructure funded by the Walloon Region under the grant agreement n1117545. Part of this work was funded by the Fonds de la Recherche Scientifique de Belgique (F.R.S.-FNRS, Mobility grant for TU-Delft). A.V. is also supported by the European Union Seventh Framework Programme (FP7/2007-2013) under a Marie Curie Career Integration Grant (grant agreement PCIG13-GA-2013-618159).

\section{References}

\section{References}

[1] DL Elliott. Status of wake and array loss research. Technical report, Pacific Northwest Lab., Richland, WA (United States), 1991.

[2] Jens N. Sørensen and Asger Myken. Unsteady actuator disc model for horizontal axis wind turbines. Journal of Wind Engineering and Industrial Aerodynamics, 39(1):139 - 149, 1992.

[3] Luis A Martinez, Stefano Leonardi, Matthew J Churchfield, and Patrick J Moriarty. A comparison of actuator disk and actuator line wind turbine models and best practices for their use. AIAA Paper, 900, 2012 .

[4] Philippe Chatelain, Stéphane Backaert, Grégoire Winckelmans, and Stefan Kern. Large eddy simulation of wind turbine wakes. Flow, Turbulence and Combustion, 91(3):587-605, Oct 2013.

[5] P Chatelain, M Duponcheel, S Zeoli, S Buffin, D-G Caprace, G Winckelmans, and L Bricteux. Investigation of the effect of inflow turbulence on vertical axis wind turbine wakes. Journal of Physics: Conference Series, 854(1):012011, 2017.

[6] Wen Zhong Shen and J Sørensen. Numerical modeling of wind turbine wakes. J. Fluids Eng, 124(2):393399, 2002.

[7] Angus CW Creech and Wolf-Gerrit Früh. Modeling wind turbine wakes for wind farms. Alternative Energy and Shale Gas Encyclopedia, pages 28-51, 2016.

[8] Robert Mikkelsen. Actuator disc methods applied to wind turbines. PhD thesis, Technical University of Denmark, 2003.

[9] N Troldborg. Actuator Line Modeling of Wind Turbine Wakes. PhD thesis, thesis DTU-MEK Denmark, 2008.

[10] Per-Åge Krogstad and Pål Egil Eriksen. "blind test" calculations of the performance and wake development for a model wind turbine. Renewable energy, 50:325-333, 2013.

[11] V. Moureau, P. Domingo, and L. Vervisch. Design of a massively parallel CFD code for complex geometries. C.R. Mecanique, 339(2/3):141-148, 2011.

[12] Matthias Kraushaar. Application of the compressible and low-Mach number approaches to Large-Eddy Simulation of turbulent flows in aero-engines. PhD thesis, Institut National Polytechnique de ToulouseINPT, 2011.

[13] A.J. Chorin. Math. Comp., 22:745-762, 1968.

[14] Mathias Malandain, Nicolas Maheu, and Vincent Moureau. Optimization of the deflated conjugate gradient algorithm for the solving of elliptic equations on massively parallel machines. Journal of Computational Physics, 238:32 - 47, 2013.

[15] V Moureau, P Domingo, and L Vervisch. From large-eddy simulation to direct numerical simulation of a lean premixed swirl flame: Filtered laminar flame-pdf modeling. Combustion and Flame, 158(7):1340-1357, 2011.

[16] Jens Nørkær Sørensen and Wen Zhong Shen. Numerical modeling of wind turbine wakes. Journal of Fluids Engineering, 124(2):393-399, 052002.

[17] Niels Troldborg. Actuator line modeling of wind turbine wakes. PhD thesis, 2009.

[18] Yulia Peet, Paul Fischer, Guenter Conzelmann, and Veerabhadra Kotamarthi. Actuator line aerodynamics model with spectral elements. In 51st AIAA Aerospace Sciences Meeting including the New Horizons Forum and Aerospace Exposition, 2013. 
[19] Klaus-Jürgen Bathe. Computational fluid and solid mechanics. Springer, 2001.

[20] Franck Nicoud, Hubert Baya Toda, Olivier Cabrit, Sanjeeb Bose, and Jungil Lee. Using singular values to build a subgrid-scale model for large eddy simulations. Physics of Fluids (1994-present), 23(8):085106, 2011.

[21] Maria-Cecilia Rivara. Mesh refinement processes based on the generalized bisection of simplices. SIAM Journal on Numerical Analysis, 21(3):604-613, 1984.

[22] Pierre Benard, Guillaume Balarac, Vincent Moureau, Cecile Dobrzynski, Ghislain Lartigue, and Yves D'Angelo. Mesh adaptation for large-eddy simulations in complex geometries. International Journal for Numerical Methods in Fluids, 81(12):719-740, 2016. 